\title{
A Method for Satisfaction Degree Decision Making with Preference Information on Criteria Weight Obtaining by Consistency Degree of Security Chip Design
}

\author{
Tao Chen, Xingguo Luo, Wei Li, Jiaxiang Gu and Ninglong Zhu \\ Zhengzhou Institute of Information and Technology, Henan Zhengzhou 450000
}

\begin{abstract}
In order to solve the problem of traditional security chip architecture design totally depending on the subjective experience decision, as while as overcoming the compatibility of multi-attribute decision completely ignored the degree of subjective experience, a method for satisfaction degree decision making with preference information on criteria weight obtaining by consistency degree is proposed, which validated the feasibility and effectiveness in architecture design combined with the security chip design evaluation.
\end{abstract}

Keywords - Consistency Degree, Satisfaction Degree, Security chip, Multiple-Attribute Decision Making

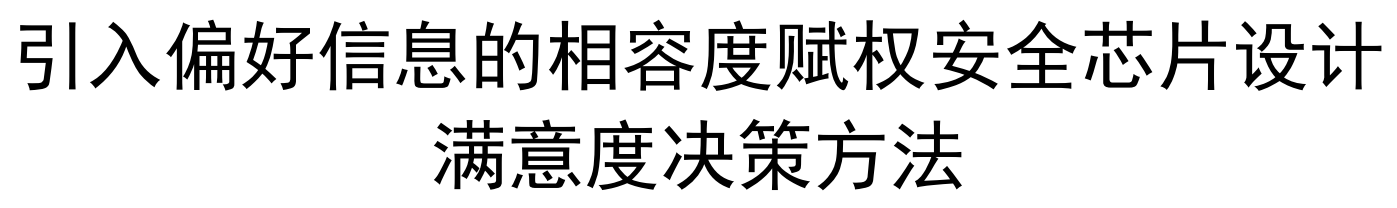

陈蹈 罗兴国 李伟 顾嘉祥 朱宁龙

郑州信息科技学院, 河南, 郑州 450000

\begin{abstract}
摘＼cjkstart要 为了解决传统安全芯片设计中完全依赖经验的体系结构主观决策带来的问题，同时克服多属性决策中相容度赋权完全忽 略主观经验判定的缺陷, 提出了一种引入偏好信息的相容度赋权方法和基于满意度选择的多属性决策方法, 结合安全芯片设计评估, 验证了该方法的可行性和有效性。
\end{abstract}

关键词 相容度, 满意度, 安全芯片, 多属性决策

1. 引言

应用驱动的安全芯片设计复杂度日益增加, 需要综合 考虑的因素也越来越复杂, 完全依赖经验的体系结构决策 容易造成所设计的安全芯片缺乏科学性与合理性。参照当 前对武器系统的作战能力评估常采用的多指标综合评价 法, 可将该问题转化为多属性决策问题 [1,2]。

多属性决策问题的核心包括两大部分: 一是决策信息 的获取, 即确定对象的属性值和属性权重, 主要有专家咨 询法、离差最大化赋权法等[1-7]。在安全芯片的设计过程 中, 决策者虽然对某些属性有一定的认知, 但对所有的属 性权重并不能精确确定, 单纯采用客观赋权法或主观赋权 法，都不能很好地解决属性权重确定的问题。

二是通过特定方法对决策信息进行处理, 使其更加直
观和具有可比性，从而对方案进行排序。现有线性加权、 层次分析法等决策方法 [1-5]中, 大部分方法均不能直接适 用于安全芯片的多属性决策过程, 如理想解法处理相差较 大的数据时, 仍可能得到距离相近的结果; 主成分析法对 于细节问题无法解决, 必须结合其他方法进行决策; 标准 水平方法和字典法适用于要求明确、过程简单的决策问题, 而安全芯片的设计选择在大部分时候要求较复杂, 涉及属 性判别因素较多; 层次分析法主要适用于层次分明的情况, 而安全芯片的某些属性间是相互交叉，相互影响的。

本文对基于相容度赋权的满意度决策过程进行了改 进, 提出了引入偏好信息相容度赋权、基于满意度的安全 芯片设计多属性决策方法, 决策过程充分结合了专家咨询 法可以充分体现专家主观意见、反映决策者的意愿和基于 
相容度的客观赋权可以充分反映数据的客观规律的特点, 通过在决策阶段引入广泛应用的线性加权法, 再结合功效 系数法的思想, 用满意度帮助决策者进行决策, 解决了安 全芯片在不同的环境下所侧重的属性不同这一要求, 能充 分反映决策者的意愿。算例表明在解决安全芯片体系结构 设计多属性决策问题时科学、有效。

\section{2. 满意度和相容度赋权相关理论}

\section{1 决策环境相关理论}

决策矩阵、决策指标及三角模糊数 [1]描述用于构成决 策环境。

定义 1: 决策矩阵, 经过对决策问题的描述（包括设 立多属性指标体系)、各指标的数据采集, 形成可以规范化 分析的多属性决策矩阵。设有 $\mathrm{n}$ 个决策指标 $f_{i}(1 \leq j \leq n)$, $\mathrm{m}$ 个备选方案 $a_{i}(1 \leq i \leq m), \mathrm{m}$ 个方案 $\mathrm{n}$ 个指标构成的矩阵 $X=\left(X_{i j}\right)_{m^{*} n}$ 称为决策矩阵。决策矩阵是规范化分析的基 础。

定义 2: 决策指标, 可分两类: 效益型（正向）指标, 数值越大越优; 成本型指标 (逆向指标), 数值越小越优。

定义 3: 三角模糊数, 称 $a=\left(a^{L}, a^{M}, a^{N}\right)$ 为三角模糊 数, 如果它的隶属函数为 $u_{a}(X): R \rightarrow[0,1], \mathrm{R}$ 为区间 $[1$, 0]上的实数集。

根据专家对象的评价选取相应的 $a^{L}, a^{M}, a^{N}$ 值, 如对指 标属性的评价可采用“极低”、“很低”、“低”、“一般”、“较 高”、“高”、“很高”、“极高”等语言变量, 语言变量和三角 模糊数间转换关系:

表 1 语言变量和三角模糊数转换关系

\begin{tabular}{|c|c|c|c|}
\hline 语言 & 三角模糊数 & 语言变量 & 三角模糊数 \\
\hline 极高 & $(0.8,0.9,1.0)$ & 较低 & $(0.3,0.4,0.5)$ \\
\hline 很高 & $(0.7,0.8,0.9)$ & 低 & $(0.2,0.3,0.4)$ \\
\hline 高 & $(0.6,0.7,0.8)$ & 很低 & $(0.1,0.2,0.3)$ \\
\hline 较高 & $(0.5,0.6,0.7)$ & 极低 & $(0.0,0.1,0.2)$ \\
\hline 一般 & $(0.4,0.5,0.6)$ & & \\
\hline
\end{tabular}
式 $^{[15]}$ :

三角模糊数可由正式近似表示或 “转化” 为区间数形

$$
\tilde{a}=\left[\boldsymbol{I}_{L}(\tilde{a}), \boldsymbol{I}_{R}(\tilde{a})\right]=\left[\frac{l+m}{2}, \frac{m+u}{2}\right]
$$

\section{2 满意度理论}

满意度 [9]的概念用来描述满意度在本文中的运用方 式。在一般的决策过程中, 决策者会对决策对象的某些属
性的属性值有一个最基本的要求或者心理可接受的预期区 间, 可称为基本期望区间 $[12,13]$, 然后根据在每个属性上 的满意度, 形成对该决策对象在该属性上的局部满意度。

1、局部满意度

假设决策者在属性 $C_{j}(j=1,2, \cdots m)$ 上的决策属性的最 低期望值为 $\tilde{A}_{j}=\left[a_{j}^{L}, a_{j}^{U}\right]$, 心理预期的最高满意度期望值为 $\tilde{B}_{j}=\left[b_{j}^{L}, b_{j}^{U}\right]$, 针对效益型指标的属性, 也就是说当决策表 中的数据在属性 $C_{j}$ 上的属性值小于或等于 $\tilde{A}_{j}$ 时, 决策者在 该属性上的的满意度为 0 ; 当属性值等于或大于 $\tilde{B}_{j}$ 时, 决 策者在该属性上的满意度为 1 ; 当属性值介于 $\tilde{A}_{j}$ 与 $\tilde{B}_{j}$ 之间 时, 决策者在该属性上的满意度呈线性变化。

成本型指标满意度函数 $\mathrm{a}$ 与效益型指标的满意度函数 $\mathrm{b}$ 的变化规律正好相反。

2、总体满意度

根据局部满意度及属性权重, 信息融合得到每个对象 的总体满意度。

定义 4：假设决策者在属性 $C_{j}(j=1,2, \cdots m)$ 上的决策 属性的权重为 $w_{j}$, 决策者根据属性值勤与心理预期区间 确定求的决策对象 $A_{i}(i=1,2, \cdots n)$ 在属性 $C_{j}$ 上的满意度为 $S D\left(\frac{A_{i}}{C_{j}}\right)(j=1,2, \cdots m)$, 那么决策者对对策对象 $A_{i}$ 的总体满 意度为 $S D\left(A_{i}\right)$, 其中:

$$
S D\left(A_{i}\right)=\sum_{j=1}^{m} S D\left(\frac{A_{i}}{C_{j}}\right) * w_{j}
$$

利用局部满意度函数 $\mathrm{a}$ 和 $\mathrm{b}$, 篮选出来达不到决策者对 满意度最低要求的对象, 对剩下的令决策者满意的决策对 象进行排列选择。

\section{3 区间数相容关系}

区间数相容关系 [9]是本文的基础, 改进方法的主要推 导过程基于该理论得出。

定义 5: 记: $\tilde{a}=\left[a^{L}, a^{U}\right]=\left\{x \mid a^{L} \leq x \leq a^{U}, a^{L}, a^{U} \in R\right\}$, 称 $\tilde{a}$ 为一个区间数, 特别的, 若 $a^{L}=a^{U}, \tilde{a}$ 退化为一个实 数。

设 $\tilde{a}=\left[a^{L}, a^{U}\right], \tilde{b}=\left[b^{L}, b^{U}\right]$, 则有以下法则:

（1） $\tilde{a} \cap \tilde{b}=\left[\max \left\{a^{L}, b^{L}\right\}, \min \left\{a^{U}, b^{U}\right\}\right]$, 当且仅当, $a^{U}>b^{L}$ 或者 $b^{U}>a^{L}$ 。

（2） $\tilde{a} \cup \tilde{b}=\left[\min \left\{a^{L}, b^{L}\right\}, \max \left\{a^{U}, b^{U}\right\}\right]$, 当且仅当, $a^{U}>b^{L}$ 或者 $b^{U}>a^{L}$ 。

定义 6: 称 $\tilde{X}^{*}=\left(\tilde{x}_{1}^{*}, \tilde{x}_{2}^{*}, \cdots, \tilde{x}_{m}^{*}\right)$ 为区间型的理想序列, 其中: 
$\tilde{X}^{+*}=\left[x_{j}^{x^{*} L}, x_{j}^{+* U}\right]=\left[\max \left(x_{i j}^{L}\right), \max \left(x_{i j}^{U}\right)\right], j=(1,2, \cdots, m)$ 为 正 理想点 (越大越好);

$\tilde{X}^{-*}=\left[x_{j}^{-*^{*}}, x_{j}^{-* U}\right]=\left[\min \left(x_{i j}^{L}\right), \min \left(x_{i j}^{U}\right)\right], j=(1,2, \cdots, m)$ 为负理 想点（越小越好）。

由正理想点构成的序列为正理想特征序列, 由负理想 点构成的序列为负理想对象。

定义 7: 设区间数 $\tilde{a}=\left[a^{L}, a^{U}\right], \tilde{b}=\left[b^{L}, b^{U}\right], T(\tilde{a}, \tilde{b})$ 是 $\tilde{a}, \tilde{b}$ 之间的相容度, 则

$$
T(\tilde{a}, \tilde{b})=\left\{\begin{array}{l}
0, a^{U}<b^{L} \text { orb } b^{U}<a^{L} \\
1, \tilde{a}=\tilde{b} \\
\frac{l_{\tilde{a} \cdot \tilde{b}}}{l_{\tilde{a} \cup \tilde{b}}}, a^{U}>b^{L} \text { orb } b^{U}>a^{L}
\end{array}\right.
$$

区间数 $\tilde{a}$ 与 $\tilde{b}$ 之间的相容度有: $0 \leq T(\tilde{a}, \tilde{b}) \leq 1$ 。两区 间数之间的共同覆盖区域与可能覆盖区域之间的比值即是 区间数相容度, 它可也表示两个区间数之间的共同信息的 重合度。

\section{3. 改进的基于相容度赋权的满意度决策方法}

\section{1 对安全芯片不同属性的规范化处理}

安全芯片的属性有多种类型, 如果仅仅用实数进行描 述, 则无法精确地反应实际情况, 属性的数据类型大致可 以分为两大类, 一是我们熟知的精确数, 二是应用越来越 多的模糊数。精确数通常即为实数, 可以对比较确定的属 性进行描述, 模糊数有多种, 在本文中选用区间数和三角 模糊数, 区间数用来描述数值不固定的属性, 如功耗、运 算速度等, 安全芯片的安全性、功能完备性等属性是语言 型变量, 不能用数值来反映, 用三角模糊数来表示。

由于数据类型的不同, 则会使决策过程变的更加复杂, 使属性间不具有可比性, 所以本文的处理方式是将不同的 数据类型转化为同一类型, 将实数看作特殊的区间数, 三 角模糊数则根据文献 ${ }^{[14]}$ 提供的公式转化为区间数, 这样就 为决策方案的实施提供了简便的前期处理方案。

\section{2 对基于相容度赋权方法的改进}

相容度描述了两个区间数之间共同信息的重合度。通 常, 当某两个方案的某个属性的重合度越大时, 说明该属 性的属性值给决策者提供的信息基本一致, 那么该属性对 于决策者来说所起的作用也较小, 从而在决策中相应的属 性权重也应该较小。相反, 当信息重合度越小时, 那么该 属性上的属性值对于决策者区分决策对象的起到的作用就
越大, 从而属性权重也应该较大。所以属性权重的大小与 属性值的相容度之间存在反比关系。

基于相容度赋权的传统方法, 符合客观规律, 能够一 定程度上反映数据的重要程度, 但仅仅考虑了数据本身的 客观规律, 并没有考虑各个属性本身的重要程度, 适用于 属性权利完全未知的情况下。而在安全芯片的设计选取过 程中, 在大部分情况下, 对某些属性是有所偏好的, 所以 在本文中引入了反映主观信息的偏好因子, 使属性的最终 权重既能反映数据客观规律, 又能反映人的偏好意愿。偏 好因子的获得，根据专家咨询法所获得的结果确定。

在加入主观的偏好因子后, 再对赋权公式重新推导, 过程如下:

对于属性 $u_{j}$, 用 $V_{i j}(w)$ 表示方案 $x_{i}$ 与其他所有方案之 间的相容度的差数, 则可定义:

$$
\begin{aligned}
V_{j}(w) & =\sum_{i=1}^{n} V_{i j}(w) \\
& =\sum_{i=1}^{n} \sum_{k=1}^{n} a_{j}\left(1-T\left(\tilde{f}\left(A_{i} / C_{j}\right) \tilde{f}\left(A_{k} / C_{j}\right)\right)\right) w_{j}, j \in M
\end{aligned}
$$

其中, 称 $\boldsymbol{a}_{j}$ 为属性 $u_{j}$ 的偏好信息。

则 $V_{j}(w)$ 表示对属性 $u_{j}$ 而言, 所有方案与其他方案的 总相容度差数。根据上述分析, 加权向量的 $\mathrm{w}$ 的选择应使 所有属性对所有方案的总相容度差数最大。为此, 构造目 标函数为

$$
\begin{aligned}
\max V(w) & =\sum_{j=1}^{m} V_{j}(w) \\
& =\sum_{j=1}^{m} \sum_{i=1}^{n} \sum_{k=1}^{n} a_{j}\left(1-T\left(\tilde{f}\left(A_{i} / C_{j}\right), \tilde{f}\left(A_{k} / C_{j}\right)\right)\right) \boldsymbol{W}_{j}
\end{aligned}
$$

于是, 求解权重向量 $\mathrm{w}$ 可通过求解如下最优化模型得 到:

$$
\left\{\begin{array}{l}
\max V(w)==\sum_{j=1}^{m} \sum_{i=1}^{n} \sum_{k=1}^{n} a_{j}\left(1-T\left(\tilde{f}\left(A_{i} / C_{j}\right), \tilde{f}\left(A_{k} / C_{j}\right)\right)\right) W_{j} \\
\text { s.t. } \quad \mathcal{W}_{j} \geq 0, j \in M, \sum_{j=1}^{m} w_{j}^{2}=1 .
\end{array}\right.
$$

作拉格朗日函数, 得到如下结果:

$$
L(w, \xi)=\sum_{j=1}^{m} \sum_{i=1}^{n} \sum_{k=1}^{n} a_{j}\left(1-T\left(\tilde{f}\left(A_{i} / C_{j}\right), \tilde{f}\left(A_{k} / C_{j}\right)\right)\right) W_{j}+\frac{1}{2} \xi\left(\sum_{j=1}^{m} w_{j}^{2}-1\right) .
$$

对该式子求偏导, 并令:

$$
\left\{\begin{array}{l}
\frac{\partial L}{\partial \mathcal{W}_{j}}==\sum_{i=1}^{n} \sum_{k=1}^{n} a_{j}\left(1-T\left(\tilde{f}\left(A_{i} / C_{j}\right), \tilde{f}\left(A_{k} / C_{j}\right)\right)\right)+\xi W_{j}=0, j \in M, \\
\frac{\partial L}{\partial \xi}=\sum_{j=1}^{m} w_{j}^{2}-1=0
\end{array}\right.
$$

得到最优解 


$$
w_{j}^{*}=\frac{a_{j} \sum_{i=1}^{n} \sum_{k=1}^{n}\left(1-T\left(\tilde{f}\left(A_{i} / C_{j}\right), \tilde{f}\left(A_{k} / C_{j}\right)\right)\right) \mathcal{W}_{j}}{\sqrt{\sum_{j=1}^{m} a_{j}\left[\sum_{i=1}^{n} \sum_{k=1}^{n}\left(1-T\left(\tilde{f}\left(A_{i} / C_{j}\right), \tilde{f}\left(A_{k} / C_{j}\right)\right)\right) W_{j}\right]^{2}}}, j \in M
$$

归一化约束条件在传统的加权向量中都是满足的, 单 位化约束条件则不作特殊要求, 以上得到的是单位化的权 重向量 $w_{j}^{*}$, 再对 $w_{j}^{*}$ 进行归一化处理, 可以使其与传统的 方法保持一致, 令

$$
w^{*}=\frac{w_{j}^{*}}{\sum_{j=1}^{m} w_{j}^{*}}, j \in M
$$

可以得到:

$$
W_{j}=\frac{a}{\sum_{j=1}^{n} \sum_{i=1}^{n} \sum_{k=1}^{n} \sum_{k=1}^{n}\left(1-T\left(\tilde{f}\left(A_{i} / C_{j}\right), \tilde{f}\left(A_{k} / C_{j}\right)\right)\right)}, j \in M
$$

当 $a_{j} \sum_{i=1}^{n} \sum_{k=1}^{n}\left(1-T\left(\tilde{f}\left(A_{i} / C_{j}\right), \tilde{f}\left(A_{k} / C_{j}\right)\right)\right)=0$ 时, 表明所有决 策对象在属性 $C_{j}$ 上的属性值是相同的, 则该属性不起任何 作用, 权重应为 0 。

\section{3 改进的基于满意度选择安全芯片的具体决策步骤}

1、对决策方案的属性进行处理, 使用表 1 所示方法用 三角模糊数表示语言型变量, 利用式(1)再将数据类型全部 转化为到区间数;

2、确定期望区间, 根据函数 a,b, 求解每个决策对象 各个属性的局部满意度, 将满意度不符合要求的决策对象 剔除;

3、利用专家咨询法 ${ }^{[1]}$ 确定各属性的偏好信息;

4、对于新的决策表中的决策对象, 利用公式(13)确定 属性权重向量 $w$;

5、对于决策矩阵进行规范化处理 ${ }^{[1]}$, 得到规范化矩阵;

6、由于前述处理的数据最终以区间数的形式给出, 一 般的排序方法不适用, 因此, 本步骤使用一种简单合理的 区间数排序方法一一属性值优势关系排序算法 [9-11], 对决 策对象进行排序;

7、用属性权重向量 $w$ 与新决策表中的决策对象在每个 属性上的满意度 $S D\left(\frac{A_{i}}{C_{j}}\right)$, 根据公式(2)确定决策对象的总 体满意度, 找出决策者满意度最大的决策对象。

\section{4. 算例和结果分析}

某研究机构打算设计一种新型安全芯片, 现在共有 8 种成品芯片体系结构参数经验值可供参考, 我们用
$U=\left\{A_{1}, A_{2}, A_{3}, A_{4}, A_{5}, A_{8}, A_{4}\right.$, 来表示, 若该单位选择时需 要考虑 $C=\left\{C_{1}, C_{2}, C_{3}, C_{4}\right\} 4$ 个指标, 主频 $C_{1}$, 核心面积 $C_{2}$, 包括抗干扰能力, 抗辐射能力, 抗攻击能力, 抗电磁干扰 能力在内的安全性 $C_{3}$, 功耗 $C_{4}$ 。显然, 指标 $C_{1}$ 和 $C_{3}$ 为效 益型指标, $C_{2}$ 和 $C_{4}$ 为成本型指标。由于功耗不是精确数, 所以用区间数表示, 安全性无法用数值度量, 所以用语言 型变量表示。

根据该单位主观意愿, 对效益型指标主频 $(\mathrm{Mhz})$, 初 步确定的期望区间为 (90,160), 安全性的期望区间为 (一 般, 高), 即仅针对主频来说, 当大于等于 160 时, 满意度 为 1 , 当主频小于等于 90 时, 满意度为 0 。对成本型指标 核心面积 $(\mathrm{mm} 2)$ 和功耗 $(\mathrm{mw})$, 对核心面积的期望面积是 $(180,400)$, 对功耗的期望值是 [ $(400,500)$ ，(800，950）]。 即仅从核心面积考虑时, 核心面积小于等于 180 时, 期望 满意度为 1 , 当核心面积大于或等于 400 时, 满意度为 0 。 直观数据如下表所示:

表 1 决策数据表

\begin{tabular}{|c|c|c|c|c|}
\hline $\mathrm{U}$ & 主频 $\mathrm{C}_{1}$ & 核心面积 $\mathrm{C}_{2}$ & 安全性 $\mathrm{C}_{3}$ & 功耗 $\mathrm{C}_{4}$ \\
\hline $\mathrm{A}_{1}$ & 100 & 150 & 高 & $(400,500)$ \\
\hline $\mathrm{A}_{2}$ & 180 & 230 & 较高 & $(600,800)$ \\
\hline $\mathrm{A}_{3}$ & 150 & 300 & 很高 & $(650,750)$ \\
\hline $\mathrm{A}_{4}$ & 130 & 360 & 一般 & $(1000,1200)$ \\
\hline $\mathrm{A}_{5}$ & 200 & 190 & 很高 & $(600,750)$ \\
\hline $\mathrm{A}_{6}$ & 130 & 410 & 极高 & $(300,450)$ \\
\hline $\mathrm{A}_{7}$ & 90 & 320 & 低 & $(500,700)$ \\
\hline $\mathrm{A}_{8}$ & 160 & 330 & 较高 & $(650,750)$ \\
\hline
\end{tabular}

步骤一: 先将语言型属性用三角模糊数表示, 再根据 公式(1)将三角模糊数转化为区间数, 得到如下结果:

表 2 语言型变量转化表

\begin{tabular}{|c|c|c|c|}
\hline & 语言型 & 三角模糊数 & 区间数 \\
\hline $\mathrm{A}_{1}$ & 高 & $(0.6,0.7,0.8)$ & $(0.65,0.75)$ \\
\hline $\mathrm{A}_{2}$ & 较高 & $(0.5,0.6,0.7)$ & $(0.55,0.65)$ \\
\hline $\mathrm{A}_{3}$ & 很高 & $(0.7,0.8,0.9)$ & $(0.75,0.85)$ \\
\hline $\mathrm{A}_{4}$ & 一般 & $(0.4,0.5,0.6)$ & $(0.45,0.55)$ \\
\hline $\mathrm{A}_{5}$ & 很高 & $(0.7,0.8,0.9)$ & $(0.75,0.85)$ \\
\hline $\mathrm{A}_{6}$ & 极高 & $(0.8,0.9,1.0)$ & $(0.85,0.95)$ \\
\hline $\mathrm{A}_{7}$ & 低 & $(0.2,0.3,0.4)$ & $(0.25 .0 .35)$ \\
\hline $\mathrm{A}_{8}$ & 较高 & $(0.5,0.6,0.7)$ & $(0.55,0.65)$ \\
\hline
\end{tabular}

由于实数可以看作特殊的区间数, 据此容易得到新的 决策表。 
步骤二: 依据成本与效益满意度函数, 按照该机构的 初始期望数值, 求解对 8 个决策对象在 4 个属性上的满意 度。由于区间数在其取值域内每个点的概率均相等, 这里 利用其期望值求解, 得到所有决策对象属性的满意度如下 所示:

表 3 满意度表

\begin{tabular}{|c|c|c|c|c|}
\hline $\mathrm{U}$ & $\mathrm{C}_{1}$ & $\mathrm{C}_{2}$ & $\mathrm{C}_{3}$ & $\mathrm{C}_{4}$ \\
\hline $\mathrm{A}_{1}$ & 0.14286 & 1 & 0.75 & 1 \\
\hline $\mathrm{A}_{2}$ & 1 & 0.77273 & 0.5 & 0.41176 \\
\hline $\mathrm{A}_{3}$ & 0.85714 & 0.45455 & 1 & 0.41176 \\
\hline $\mathrm{A}_{4}$ & 0.57143 & 0.18182 & 0.25 & 0 \\
\hline $\mathrm{A}_{5}$ & 1 & 0.95455 & 0.75 & 0.47059 \\
\hline $\mathrm{A}_{6}$ & 0.57143 & 0 & 1 & 1 \\
\hline $\mathrm{A}_{7}$ & 0 & 0.36364 & 0 & 0.64706 \\
\hline $\mathrm{A}_{8}$ & 1 & 0.31818 & 0.5 & 0.41176 \\
\hline
\end{tabular}

设定决策者心理的期望值确定的满意度大于 0 时, 所 有的决策对象均进入考虑范围, 有 $\{\mathrm{A} 1, \mathrm{~A} 2, \mathrm{~A} 3, \mathrm{~A} 5$, A8\} 5 个芯片可供选择, 得到新的决策表 2

表 4 决策数据表 2

\begin{tabular}{|c|c|c|c|c|}
\hline $\mathrm{U}$ & $\mathrm{C}_{1}$ & $\mathrm{C}_{2}$ & $\mathrm{C}_{3}$ & $\mathrm{C}_{4}$ \\
\hline $\mathrm{A}_{1}$ & $(100,100)$ & $(150,150)$ & $(0.65,0.75)$ & $(400,500)$ \\
\hline $\mathrm{A}_{2}$ & $(180.180)$ & $(230,230)$ & $(0.55,0.65)$ & $(600,800)$ \\
\hline $\mathrm{A}_{3}$ & $(150,150)$ & $(300,300)$ & $(0.75,0.85)$ & $(650,750)$ \\
\hline $\mathrm{A}_{5}$ & $(200,200)$ & $(190,190)$ & $(0.75,0.85)$ & $(600,750)$ \\
\hline $\mathrm{A}_{8}$ & $(160,160)$ & $(330.330)$ & $(0.55,0.65)$ & $(650,750)$ \\
\hline
\end{tabular}

步骤三: 假设该单位经过运用专家咨询法判定的各属 性的主观权重如下表所示:

表 5 主观权重表

\begin{tabular}{|c|c|c|c|c|}
\hline $\mathrm{U}$ & $\mathrm{C}_{1}$ & $\mathrm{C}_{2}$ & $\mathrm{C}_{3}$ & $\mathrm{C}_{4}$ \\
\hline $\mathrm{w}$ & 0.24 & 0.23 & 0.28 & 0.25 \\
\hline
\end{tabular}

步骤四: 运用属性值赋权公式与表中的数据可以求得 各个属性的权重为: $\mathrm{W} 1=0.28506, \mathrm{w} 2=0.27319$, w3 $=0.26606, w 4=0.17569$ 。

步骤五、六: 对决策矩阵进行规范化处理, 得到规范 化矩阵, 用属性值优势关系算法[11]对决策对象进行排序, 得到结果为:

表 6 常规排序表

\begin{tabular}{|c|c|c|c|c|}
\hline $\mathrm{A} 1$ & $\mathrm{~A} 2$ & $\mathrm{~A} 3$ & $\mathrm{~A} 5$ & $\mathrm{~A} 8$ \\
\hline 1.1199 & 0.93617 & 0.9074 & 1.371 & 0.51861 \\
\hline
\end{tabular}

即: $\mathrm{A} 5>\mathrm{A} 1>\mathrm{A} 2>\mathrm{A} 3>\mathrm{A} 8$
由此结果可得出, A5 为最优选择方案。

步骤七: 计算各个方案的总体满意度, 结果为:

表 7 总体满意度表

\begin{tabular}{|c|c|c|c|c|}
\hline $\mathrm{A} 1$ & $\mathrm{~A} 2$ & $\mathrm{~A} 3$ & $\mathrm{~A} 5$ & $\mathrm{~A} 8$ \\
\hline 0.68914 & 0.70154 & 0.70692 & 0.82805 & 0.57736 \\
\hline
\end{tabular}

\section{即: $\mathrm{A} 5>\mathrm{A} 3>\mathrm{A} 2>\mathrm{A} 1>\mathrm{A} 8$}

此时, 满意度最高的决策对象与最优决策对象均为 A5 根据该算例可以看出, 用优势关系算法得到的排序结 果与用本文得到的排序结果不一样, 表明满意度反映的信 息与客观排序方法有差别, 从而可以为决策者提供有效的 决策信息。在某些情况下, 如果两者最优的决策对象不一 致, 则要根据实际情况慎重考虑, 作出合理的判断。

\section{5. 小结}

本文研究了安全芯片设计中的多属性决策问题, 采用 区间数规范化安全芯片各种属性, 以主观与客观相结合的 方法来确定属性权重, 用满意度帮助决策者进行最终决策, 为决策者提供了一种新的决策方法。文中, 满意度的确定 方式是简单线性的赋值, 与实际情况不完全相同, 还有待 改进, 下一步将通过对客观权重与主观偏好信息结合方式 的试验, 进一步研究其结合方式, 以确定更合理的主客观 结合方式。

\section{参考文献(References)}

[1] Xu Zeshui, Uncertain Multiple Attribute Decision Making: Methods and Applications. Beijing, Tsinghua University Press, 2004

[2] Xu Jiuping and $\mathrm{Wu}$ Wei, Multiple Attribute Decision Making Theory and Methods, Beijing, Tsinghua University Press, 2006

[3] Yu Yan and Liang Liang, Futher Investigation on TOPSIS Method with Multi-Target Strategic Decision, Systems Engineering, 2003, 21(2):98-101

[4] Wang Yingchao, Sun Hongyue, Shang Jiquan and Zhang Yun, Application of Efficacy Coefficient Method to Instability Risk Early-Warning of Tunnel Surrounding Rock. Chinese Journal of Rock Mechanics and Engineering, 2010.29(9):3679-3684.

[5] Li Chunping, Yang Yimin and Ge Yingyu, A Comparison on the Quantitative Appraisal of Multi-index Between the Principal Component Analysis Algorithm and the Analytical Hierarchy Process. Journal of Nanjing University of Finance and Economics, 2005.136(6):54-57.

[6] Luo Dang, An Eigenvector Method for Grey Decision-making. Systems Engineering-theory \& Practice, 2005.4:67-71.

[7] Zhou Xuan, Zhang Fengming, Hui Xiaobin and Li Kewu, Method for determining experts' weights based on entropy and cluster analysis. Control and Decision, 2011, 26(1):153-156. 
[8] Wang, Y.M., Lui, Y..Integration of correlations with standard deviations for determining attribute weights in multiple attribute decision making. Mathematical and Computer Modelling, 2010, 51(1-2):1-12.

[9] Liu Jian, Liu Sifeng, Zhou Xianzhong and Xue Li, Research on the Satisfaction Degree and Criteria W eight Obtaining for Multiple Criteria Decision Making Problem. Chinese Journal of Management Science, 2011, 19(6):126-130.

[10] Liu Jian and Liu Sifeng, Research on the Ranking of Multiple Decision Object for Attribute Value within Interval Numbers. Chinese Journal of Management Science, 2010, 18(3): 90-94.

[11] Liu Jian, Xue Li, Liu Sifeng and Feng Qilei, Research on
Multiple-Attribute Decision Making Problems Based on The Superiority Index. Control and Decision, 2010, 25(7):10791087.

[12] Wang Jianqiang and Ren Shichang, Grey Random Multi-Criteria Decision-Making Approach Based on Expected Value. Control and Decision, 2009, 24(1):39-43.

[13] Yuan Guoqiang, A General Two-Stage Fizzy Production Planning Expected Value Model. Acta Mathematicae Applicatae Sinica, 2009, 32(4):648-663.

[14] Liou T S. Ranking fuzzy numbers with integral value. Fuzzy Sets and Systems 1992, 50:247-255. 